\title{
LE TRANSCENDANTAL ET LE NATURALISME: UNE RELECTURE DE KANT ET HUSSERL
}

\section{JEAN-DANIEL THUMSER}

$\mathrm{PhD}$ in Philosophy.

Ecole Normale Supérieure, Husserl-Archive.

75005 Paris, France.

E-mail: thumser.jd@hotmail.fr

\section{TRANSCENDENTALISM AND NATURALISM: A REREADING OF KANT AND HUSSERL}

This paper aims to highlight the fact that Kantian philosophy and phenomenology do not only tend to disclose the structures of a priori knowledge. Contrariwise, we suggest that the transcendental may also be understood by a detour towards the empirical. Our first example concerns Kant as he developed a certain prefiguration of the phenomenological considerations and described a way to consider the subject in a naturalistic way by introducing the notion of Selbstsetzung in his latest work. By comparing the way Kant and Husserl thought the need to consider that the subject is embodied in the world, we also point out that Husserl improved the Kantian problematic of the Selbstsetzung as he described a transcendental and scientific method to apprehend the life of the subject. Nevertheless, this method may also be defined as a "phenomenological naturalism" in so far as the questions Husserl brings to light can be seen as the seeds of the current attempt to naturalize phenomenology. In this context, we aim to demonstrate that Kant's first comprehension of the Selbstsetzung prefigures the development of the phenomenological turning point from a static to a genetic method. Furthermore, this genetic method may be conceived as an original form of naturalism which is able to illuminate the current cognitive sciences by focusing on the first-person perspective and on crucial themes like the flesh, consciousness, etc. Eventually, we conclude that this turning point may have been an inspiration for the naturalization of phenomenology.

Key words: Phenomenology, naturalism, Kant, Husserl, transcendental, practical philosophy, epistemology.

(C) JEAN-DANIEL THUMSER, 2018 


\title{
ТРАНСЦЕНДЕНТАЛИЗМ И НАТУРАЛИЗМ: ПЕРЕЧИТЫВАЯ КАНТА И ГУССЕРЛЯ
}

\author{
ЖАН-ДАНИЭЛЬ ТУМЗЕР
}

Доктор философии.

Высшая нормальная школа, Архив Гуссерля.

75005 Париж, Франция.

E-mail: thumser.jd@hotmail.fr

Цель этой статьи - продемонстрировать, что кантовская философия и феноменология не только направлены на раскрытие структур априорного знания. Напротив, мы полагаем, что трансцендентальное может быть понятно окольным путем через эмпирическое. Наш первый пример связан с Кантом, поскольку он создал известный прообраз феноменологических исследований и описал натуралистический способ рассмотрения субъекта, введя понятие Selbstsetzung в своем позднейшем труде. Сравнивая способ, которым Кант и Гуссерль продумывали необходимость рассматривать субъекта с точки зрения его телесного бытия в мире, мы также обращаем внимание на то, что Гуссерль усовершенствовал кантовскую проблематику Selbstsetzung, описав трансцендентальный и научный метод понимания жизни субъекта. Тем не менее, этот метод может быть также определен как «феноменологический натурализм» в той мере, в какой поднимаемые Гуссерлем вопросы могут рассматриваться как исходные пункты современной попытки натурализации феноменологии. В этой связи мы намерены показать, что исходное кантовское понимание Selbstsetzung предвосхищает феноменологический поворот от статического к генетическому методу. Более того, этот генетический метод может быть понят как оригинальная форма натурализма, которая способна прояснить современные когнитивные науки, фокусируясь на перспективе первого лица и таких ключевых темах, как тело, сознание etc. В конце статьи делается вывод о том, что этот поворотный пункт может служить стимулом для натурализации феноменологии.

Ключевые слова: Феноменология, натурализм, Кант, Гуссерль, трансцендентальное, практическая философия, эпистемология.

\section{INTRODUCTION}

Kant et Husserl ont tous deux développé une philosophie dite transcendantale. Lapport de tels projets est conséquent, car il permet de saisir à la fois les cadres $a$ priori à l'origine de la formation du savoir et, dans le cas de Husserl, la portée d'une reconquête du sens à partir d'un retour vers l'ego meditans. Or, dans cette optique épistémologique de fondation ultime de toute forme de connaissance, ces auteurs ont tous deux opéré un revirement conséquent dans leur philosophie qui a mené à considérer le sujet, non plus comme une forme logique et impersonnelle, mais comme un être situé et incarné. Kant, le premier, a mis au point une philosophie critique qui avait pour fin de décrire de quelle façon le sujet transcendantal accorde du sens au monde, 
tout en mettant en avant la frontière infranchissable qu'il y a entre lui et le domaine du phénoménal. Pourtant, cette forme de transcendantalisation trouve ses limites dans l'élaboration des notions d'autoposition (Selbstsetzung) et d'autoaffection lesquelles suggèrent que le sujet est un être situé dans le monde, un être qui s’auto-positionne en son sein. Il s'agit là d'une forme embryonnaire de naturalisme qui vise à comprendre le sujet comme un être au monde, c'est-à-dire à reconnaître le sujet dans sa mondanéité. Ce revirement, nous le voyons également dans la phénoménologie développée par Husserl, en particulier après la publication du premier volume des Ideen, lequel demeure essentiellement statique et kantien. Le tournant dit " génétique » de la phénoménologie est crucial, car il perpétue ce même besoin de recourir à une analyse qui ne soit pas strictement transcendantale, mais qui promeut également la vie du sujet en ses dimensions concrètes. Ce faisant, la phénoménologie opère un mouvement pareil à celui de la philosophie kantienne, mais demeure néanmoins problématique, car elle ne prend que peu en compte la dimension strictement naturelle du sujet. Est-ce ainsi possible de décrire de tels revirements comme des preuves d'une naturalisation embryonnaire ? Rappelons que la thèse de la naturalisation est que « le domaine entier de la nature, y compris la nature humaine, ne comprend que des entités et processus susceptibles d'une [...] analyse quantitative " (Cobb-Stevens, 1998, 217). Or ce type de naturalisation est largement remis en question par Kant et Husserl, ce qui contredit à première vue toute tentative d'établir un lien entre leur philosophie et toute entreprise naturaliste. Pourtant, en ayant pour objectif de décrire ce besoin de développer une philosophie qui ne pratique pas une transcendantalisation exacerbée de la vie du sujet, nous souhaitons montrer que Kant et Husserl ont tous deux posé les jalons de lactuelle de naturalisation de la phénoménologie. En particulier, nous suggérons que les textes tardifs de Husserl sont empreints d'un certain «naturalisme phénoménologique » en ce qu'ils portent sur le problème de la nature entendu non pas en des termes scientifiques, mais en un sens phénoménologique, du fait qu'il nous procure une lecture renouvelée de la notion de vie. Nous tenterons dès lors de mettre en lumière l'apport de ces deux philosophies en ce qui concerne la proposition d'une naturalisation de la phénoménologie qui soit pleinement phénoménologique. Nous aurons ainsi l'occasion de revenir sur certains points ambivalents de la pensée kantienne et de la phénoménologie, pour mettre en avant le fait que ces pensées ne portent pas atteinte aux entreprises visant une naturalisation de même qu'une science globale de la vie subjective, mais viennent les compléter. Nous espérons ainsi démontrer que la question du transcendantal va de pair avec la question du naturalisme dans le cadre de la philosophie kantienne et de la phénoménologie husserlienne en ce qu'elles rendent possible une étude cogénérative. 


\section{UN REVIREMENT PHENOMENOLOGIQUE ET NATURALISTE DANS L'OPUS POSTUMUM DE KANT ?}

En ayant pour fin d’attribuer une primauté au sujet épistémique, Kant s’applique à décrire les cadres a priori permettant à celui-ci d’apprécier et de concevoir une chose. Il fait pour cela appel aux formes pures de l'intuition que sont l'espace et le temps, de même quaux catégories de l'entendement qui sont des fonctions logiques et des représentations des choses. C'est au travers de ces formes pures et de ces catégories, pures ou en lien avec le sensible, que le sujet est capable de distinguer les divers éléments qui se présentent à lui et d'acquérir une connaissance de la chose visée sous une forme unifiée. Néanmoins un tel examen des cadres a priori, loin dêtre un exercice psychologique d'introspection, requiert de ne situer le fondement de la connaissance ni en dehors du sujet transcendantal ni à partir des qualités physiques du sujet, sous peine de perpétuer tantôt un cartésianisme suranné, tantôt un empirisme réductionniste qui réduirait chacun des vécus à des faits. L'impératif épistémologique du criticisme kantien est de trouver le fondement apodictique d'une connaissance pour garantir une science qui prenne appui sur un fondement indubitable. Or ce fondement doit puiser sa source dans le sujet qui se définit dès lors comme denkendes Ich, sujet pensant, dont la particularité est de ne pas être uniquement considéré comme moi empirique, dont on peut attester l'existence durant l'expérience sensible quotidienne à la manière de tout autre objet intramondain, mais aussi d'inclure en soi une sphère primitive qualifiée de moi pur (reine Ich) appréhendé par déduction: « Kant soutient qu’on ne saurait faire l'économie d'un sujet de l'expérience potentiellement conscient de soi-même. Il qualifie ce sujet de "transcendantal", car il ne saurait être appréhendé par la conscience empirique ni étudié par les sciences empiriques. En outre, l'accès à la dimension transcendantale s'avère toujours d'ordre déductif, plutôt qu'intuitif, puisqu'il n'y a pas d'intuitions non sensibles » (Cobb-Stevens, 1998, 197). Cette instance originaire est le principe premier dont rien ne peut être dit, sinon qu'il « doit nécessairement pouvoir accompagner toutes mes représentations » (Kant, 2006, 198). Non hypostasié, le Je kantien nest ni une substance ni un élément réal. Contrairement à Descartes, Kant reprend ainsi l'ego cogito afin d'y déceler une unité première et synthétique, qui, dépossédée de tout ancrage mondain, est présente sans pour autant être une chose empirique. Il s'agit là d'un principe originaire et synthétique que Kant nomme l'aperception pure, c'est-à-dire l'unité transcendantale ou la conscience de soi pure à partir de laquelle toute connaissance est possible. En ce sens, Kant se distingue de Descartes et nous trouvons dans les Paralogismes, en loccurence lorsqu'il traite de l'argument de la psychologie rationnelle, l'expression de son opposition radicale avec 
la position cartésienne. Il y souligne l'illusion qui a poussé la tradition néo-cartésienne à hypostasier à tort l'ego :

La proposition : Je suis simple doit être considérée comme une expression immédiate de l'aperception, tout comme le prétendu raisonnement de Descartes : cogito, ergo sum, est en fait tautologique, dans la mesure où le cogito (sum cogitans) énonce immédiatement l'effectivité. [...] Mais il est manifeste que le sujet de l'inhérence est indiqué [...] sans qu’en soit remarquée la moindre propriété [...]. Il signifie un quelque chose en général (sujet transcendantal) dont la représentation doit être absolument simple précisément parce que l'on n'y détermine rigoureusement rien. (Kant, 2006, 368)

Kant reprend ainsi le raisonnement de Descartes afin de montrer le caractère tautologique, mais surtout perlocutoire de son énoncé: « le cogito énonce immédiatement l'effectivité ». Il affirme en conséquence et sans détour que la présence du Je n'est pas seulement nécessaire, mais permanente, et avant tout qu'il n'y a aucune connaissance possible du Je en-dehors de la simple énonciation de cette effectivité. Si connaissance il y avait du moi, " des jugements synthétiques a priori seraient possibles en dehors de toute expérience » (Philonenko, 1969, 240), or cela n'est pas le cas. Le Je ne doit pas seulement pouvoir accompagner chacune des représentations, il les accompagne toutes comme point-origine, mais nest pas une représentation comme les autres ni même l'objet d'une quelconque connaissance. Inconnu à lui-même, le Je kantien n'est qu'un principe. Mais ce qui est certainement le plus notable dans cette optique est l'opacité du Je qui s'illustre le mieux lorsqu'il est question de l'" inconscient " - thème similaire à la synthèse passive dont fait état Husserl. En effet, l'une des principales difficultés relatives à la thèse kantienne du Je tient en cela que les représentations accompagnées nécessairement par le Je ne peuvent toutes être limpides. Victime d'illusions, de représentations pour le moins confuses et de pulsions, l'expérience du sujet épistémique est soumise à un enchaînement d'événements disparates. Kant va jusqu’à affirmer, non sans esprit de paradoxe, que c'est « le champ des représentations obscures qui, en l'homme, est le plus vaste " (Kant, 1993, 63), mais cela n'empêche nullement que ce même champ ne puisse être éclairci par la suite. Lors de telles représentations le Je n'est ni tout à fait absent ni tout à fait présent. Il serait plus adéquat de dire qu'il s’agit d'une « conscience médiate d'une représentation, sans que nous en soyons immédiatement conscients " (Kant, 1993, 62). Ainsi le Je, en tant qu'unité logique permettant la synthèse du divers de l'intuition, accompagne nécessairement toutes mes représentations. À la manière d'un éveil, chaque expérience peut par la suite être recouverte. En effet, l'inconscient kantien est une absence d'attention qui peut être recouverte par une conversion attentionnelle, en un sens similaire à l'activité de la synthèse passive mise en lumière par Husserl, laquelle nous permet 
dêtre toujours confrontés à des objets sans pour autant que nous soyons attentifs à tel ou tel objet. Laperception pure (apperceptio) ou transcendantale du moi, distincte de l'aperception empirique, s'illustre ainsi comme principe formel qui, par-delà toute expérience distincte ou obscure, prospère et persiste. Le Je, quoique non substantiel, subsiste quel que soit le rapport conscientiel. En somme, le moi n'est pour Kant « rien de plus que le sentiment d'une existence sans le moindre concept et il est seulement la représentation de ce à quoi se rapporte toute pensée (relatione accidentis) » (Kant, 2012, 136), un moi sans qualités.

Présent et nécessaire afin de maintenir et de garantir l'unité du divers des représentations, le moi pur kantien se révèle pas être un objet de l'expérience, bien qu'il en soit le principe unificateur. Si nous pouvons en avoir un sentiment d'existence, il n’en résulte aucun prédicat. Le moi est l'instance prédicative dont nulle chose ne peut être prédiquée. "Il signifie un quelque chose en général (sujet transcendantal) dont la représentation doit être absolument simple précisément parce que l'on n'y détermine rigoureusement rien, puisque, de fait, rien ne peut assurément être représenté avec davantage de simplicité qu’à travers le concept d'un pur quelque chose » (Kant, 2006, 368). Ainsi en arrivons-nous à une compréhension pour le moins singulière du sujet. Déchiré entre une approche transcendantale et une approche psycho-physiologique, le moi ne saurait être défini sans ambiguité, mais il trouve une ultime définition dans les écrits tardifs de Kant, en particulier dans l'Opus Postumum où, plus encore que dans la Critique de la raison pure et les Prolégomènes, l'auteur opère un revirement dans sa pensée initiale et affirme que le sujet, lors de l'aperception transcendantale se perçoit tel un Object, c'est-à-dire comme chose en soi, et non comme un objet à portée de main, un vis-à-vis (Gegenstand) :

La conscience de soi-même (apperceptio) est un acte par lequel le sujet se fait de façon générale objet (Object). Ce n'est pas encore une perception (apprehensio simplex), c'est-àdire pas une représentation sensible [...], mais une pure intuition, qui, sous l'appellation d'espace et de temps, contient simplement le côté formel de la composition (coordinatio et subordinatio) du divers de l'intuition et qui contient par là un principe a priori de la connaissance synthétique de celui-ci, mais qui précisément pour cela représente l'objet dans le phénomène. (Kant, 1986, 287)

Comme nous pouvons le noter, cette nouvelle caractérisation de Je ne signifie pas pour autant que le Je soit saisi sur le mode d'un objet mondain. La définition du terme Object peut éclaircir ce dernier point : «[...] le terme catégorial latin Object est employé pour désigner la chose en elle-même, tandis que le terme Gegenstand est employé pour la relation phénoménale, laquelle est un rapport au sujet, Subject » (Kant, 1986, 330). Cela nous laisse penser que la saisie du Je à travers une pure intuition nous 
permet d'appréhender le Je en sa dimension d'entité non mondaine, ce qui signifie de la même façon que le Je nest plus alors un simple X logique, mais un quelque chose à partir duquel l'unité formelle de la vie phénoménale puise sa source. Somme toute, l'objet-sujet que désigne ici Kant est une instance originaire à partir duquel le sujet peut se connaître dans sa relation au monde. Ainsi, en tant qu'instance formelle, le Je kantien n'est autre chose qu'un principe synthétique, logique et fonctionnel - conditio sine qua non de toute compréhension de la nature et plus généralement de tout jugement. Voilà l'objectif principal de Kant : mettre au jour les cadres a priori de la connaissance, ce qui laisse en suspens la question d'une définition du moi, de ses caractéristiques ontiques et, par conséquent de la vie du sujet. Le transcendantal semble ainsi être une impasse à une philosophie de la vie. Mais, comme le souligne pertinemment Jocelyn Benoist, l'entreprise kantienne ne consiste pas à nier qu'il puisse y avoir un moi, puisqu'il est partout en tant que forme logique principielle : « Le moi nest en un sens rien pour moi, mais en un autre sens il est partout, car il n'est rien qui puisse être pour moi sans avoir un rapport nécessaire a priori à ce moi, contenu dans le "Je pense" » (Benoist, 1994, 53). Il en ressort que Je kantien n'est pas tant un sujet ou un agent qu'un principe synthétique. Il ne serait donc pas un corps parmi les autres, mais le principe des principes ; autrement dit, un pôle vide ou, tout au plus, un point de fuite. L'entreprise kantienne serait dès lors archéologique en ce qu’elle met en lumière le principe logique originel. Aussi, en nous fiant à l'analyse quen donne Gaston Berger, le Je kantien ne peut être compris autrement qu'en tant qu' "acte logique », y compris dans l'Opus postumum :

Ainsi le sujet n'est pas éprouvé ; il est admis. Il n’est qu'une condition formelle. L'Opus
postumum lui-même, dont les tendances idéalistes sont incontestables (soit quelles tra-
duisent une intention profonde du système, soit quelles manifestent un déplacement de
point de vue sous l'influence des critiques), conserve au Je son caractère logique. Le « Je
pense ", forme universelle, a seulement perdu l'allure d'une donnée statique qu'il avait
dans la Critique, pour prendre celle « d'un acte et d'un devenir ", mais c'est encore un acte
logique. (Berger, 1941, 127-128)

Or, si Gaston Berger soutient que le Je conserve son caractère logique, d'autres lectures sont toutefois possibles. L'on pourrait considérer en effet que, contrairement à ce que ce passage laisse suggérer, Kant ait entrepris une véritable refondation de la philosophie critique dans l'Opus Postumum en voulant incarner le sujet dans le monde - aspect qui était négligé jusqu’alors comme le relève justement Michaël Foessel : "Ignorant de la réduction phénoménologique, le criticisme l'est donc aussi de la conscience absolue comme origine du monde et le "Je pense" se voit réduit à nêtre qu'une fonction d'accompagnement des actes cognitifs de l'entendement " 
(Foessel, 2015, 33). Ce revirement préfigure une nouvelle façon de concevoir l'idéalisme transcendantal en insérant le sujet dans le monde comme agent, comme sujet d'une expérience possible, en tant que le sujet se pose lui-même et est auto-affecté. Kant, en effet, met alors l'accent sur l'expérience du sujet et va même jusquà définir la philosophie transcendantale comme une possibilité pour le sujet d'être l'auteur de soi-même : "La philosophie transcendantale contient les principes synthétiques de l'intuition des choses et du penser ; espace et temps comme phénomènes, non comme agrégats, mais comme principes formels de l'unité des principes du sujet, dêtre auteur de soi-même » (Kant, 1986, 235)! Plus encore, il va jusquà caractériser la philosophie transcendantale comme « la doctrine de la sagesse qui soriente toute à ce qui est pratique dans le sujet " (Kant, 1986, 234). Plusieurs philosophes, comme Patočka ou Petit, ont également souligné cette avancée cruciale dans le développement de la pensée kantienne. Il s'agit ni plus ni moins d'une première caractérisation de la corporéité au sein de l'idéalisme transcendantal. Patočka notait à ce propos qu'il y a dans l'Opus Postumum une " aspiration à faire accorder à la corporéité le statut d'une condition a priori de la conscience pensante ». Malheureusement, ce revirement n’a pas été mené à son terme : "Cette idée, extrêmement importante et qui anticipe certains motifs les plus modernes de la phénoménologie actuelle, n’est pas, chez Kant, menée à sa conclusion " (Patočka, 2016, 188). Mais, bien que cette entreprise demeure sous une forme embryonnaire, nous pouvons noter avec Jean-Luc Petit qu'une certaine préfiguration d'une naturalisation au sens d'une inscription corporelle et mondaine du sujet transcendantal viendrait répondre à la position statique adoptée dans la Critique de la raison pure dans laquelle seules les facultés du sujet sont prises en compte. En effet, la prise en compte de l'autoaffection et de l'autodétermination du sujet revient à comprendre que celui-ci n'est pas uniquement une forme logique, mais un être situé dans le monde en interaction avec l'ensemble de l'environnement dans lequel il se trouve. Cela importe d'autant plus que Kant met l'accent sur les principes moteurs des corps dans divers passages ; le sujet, plus qu'un X logique, est un Object qui s'affecte lui-même et se représente pour apparaitre (Kant, 1986, 103). Voilà en quoi Petit peut affirmer sans difficulté la chose suivante :

En revanche, dans l'Opus postumum est esquissée une théorie de l' « autoposition » (Selbstsetzung) du sujet comme objet d'expérience [...]. On peut [...] y discerner un premier essai de "naturalisation du transcendantal ", ou au moins la preuve de ce que la philosophie transcendantale a rencontré dès l'origine l'exigence de sa naturalisation. Toute connaissance et en général toute expérience s'accompagnent d'un « je pense » qui est l'acte unifiant le divers de cette expérience en l'unité d'un objet. Mais quelle expérience pouvons-nous donc avoir d'une pure fonction d'unité logique, formelle, ou analytique, l'unité d'une diversité sensorielle quelconque? (Berthoz \& Petit, 2006, 100) 
Ces commentateurs renversent ainsi la problématique précédente en avançant que la place du sujet transcendantal doit comprendre une dimension ontique, sinon des possibilités d'effectuations (Leistungen) au sein du monde. En d'autres termes, l'idéalisme transcendantal kantien porterait en soi le germe d'une certaine naturalisation qui, étrangère à tout réductionnisme naturaliste, postule toutefois la nécessité d'un ancrage corporel et par conséquent mondain du sujet au sein même de son environnement (Umwelt). «La philosophie transcendantale est la faculté du sujet s'auto-déterminant par le complexe systématique des idées qui font un problème de la détermination complète de celui-ci comme objet (Object) (l'existence de celui-ci) pour le constituer lui-même comme donné dans l'intuition. Tout comme se faire soi-même » (Kant, 1986, 232-233). En ce sens, la philosophie kantienne tardive est notamment une anticipation des problématiques relatives à la phénoménologie husserlienne tant elle se préoccupe de l'inscription corporelle du sujet dans un monde au sein duquel il se tient et auquel il attribue du sens. Perdre de vue la dimension corporelle c'est, en effet, non seulement nier l'origine de la pensée comme pensée d'un sujet toujours déjà incarné dans le monde, mais c'est aussi mettre sous silence le caractère fondamentalement phénoménologique du corps comme lieu irréductible (Nullpunkt) au sein duquel la vie jaillit et prend forme: «Un corps organique (articulé) est celui dans lequel chaque partie avec sa force motrice se rapporte nécessairement au tout, à chaque partie de sa composition. La force productive de cette unité est la vie » (Kant, 1986, 48). Nous retrouvons cette interrogation sous une forme similaire dans le corpus phénoménologique lorsque Husserl se défait d'une approche strictement statique inspirée de l'entreprise kantienne pour développer une phénoménologie dite génétique qui met en avant la dimension expérientielle du vécu. Or, tel que nous le verrons, le tournant génétique de la phénoménologie, qui est pour ainsi dire une perpétuation du dessein kantien tardif d'une autoposition et d'une inscription corporelle du sujet au sein du monde, dépasse le cadre d'une recherche des invariants eidétiques et préfigure de la sorte ce que nous nommerons un «naturalisme phénoménologique » qui peut, selon nous, répondre à l'exigence d'une véritable philosophie scientifique appliquée à la vie consciente.

\section{HUSSERL ET LE RISQUE D'UNE TRANSCENDANTALISATION EXACERBEE DE LA VIE}

La particularité de l'entreprise husserlienne est de développer une phénoménologie de la vie du sujet qui ne porte pas exclusivement sur la sphère transcendantale. Husserl se devait de dépasser la position statique qui était la sienne jusqu’aux Ideen I 
afin que la phénoménologie puisse devenir une philosophie de la vie concrète. Ce faisant, la phénoménologie propose une analyse fine du rapport entre le Je pur, la chair et le corps matériel qui sont autant d'hypostases d'une même réalité, l'ego. Mais, plus que cela, la phénoménologie est porteuse d'un certain naturalisme phénoménologique qui sest développé depuis son tournant génétique aux environs des années 1920 et elle peut, dans une certaine mesure, venir en aide aux sciences de la nature en ce qu'elle permet d’outrepasser le fossé explicatif constaté entre les vécus intimes et létude de la conscience d'un point de vue neurophysiologique. En effet, l'analyse du vécu développée par Husserl dès le tournant génétique représente à notre sens une modalité essentielle dans le cadre d'une naturalisation de la phénoménologie, car elle représente une limite à une entreprise de transcendantalisation exacerbée de la vie et parce quelle justifie une interprétation nouvelle de la vie dans un cadre bivalent. La phénoménologie instruit ainsi les sciences cognitives en ce qu'elle met en lumière l'importance de la vie subjective analysée dans une perspective à la première personne. Le transcendantal se lie dès lors à l’ontique pour donner à une approche cogénérative. Il ne s'agit donc pas de faire de la phénoménologie une science auxiliaire des neurosciences, au contraire. Néanmoins, une approche strictement transcendantale est limitative pour plusieurs raisons.

D’abord, selon les termes de Natalie Depraz, « décrire l'ego à partir de sa dimension d'essence invariante, c'est pour Husserl mettre hors-jeu toute conception du sujet en termes de "je" particulier caractérisé par ses états internes provisoires " (Depraz, 2015, 121). En ce sens, la recherche d'invariants universels mène à une dépersonnalisation de l'ego et empêche de saisir sa vie singulière. À ce stade, la phénoménologie demeure essentiellement une méthode à la troisième personne. "L'ego en troisième personne est un Je objectivé, structurellement invariant et anonyme (un Je générique). Il est tout le monde et personne, partout et nulle part " (Depraz, 2015, 127). Laspect expérientiel et singulier de la vie du sujet semble alors être mis sous silence, tout comme il létait dans la philosophie kantienne. Or, Natalie Depraz nous fournit quant à cette problématique de précieux outils pour appréhender l'usage du Je en phénoménologie. En effet, elle propose dans Première, deuxième, troisième personne de comprendre en quoi « la première personne pourra se manifester à plein à travers une situation, un cadre spatio-temporel concret où le "je" en tant que pronom personnel peut être absent, mais l'incarnation en acte maximale » (Depraz, 2015, 135). Sa visée est une pratique phénoménologique qui met l'accent sur la part expérientielle du sujet, cette part pouvant être illustrée sans faire usage du pronom personnel « Je ». Elle retrouve une telle pratique dans le premier volume des Ideen, en particulier lorsque Husserl donne des exemples concrets, entre autres la galerie d'art à Dresden. Le « Je » y 
est fréquemment absent, mais l'incarnation et la description de l'expérience sont bien présentes. «Au fond, ajoute-t-elle, les différentes composantes d'une phénoménologie en/de la première personne (pronom personnel "je", présence du corps, inscription dans une situation spatio-temporelle concrète) deviennent actives et opératoires dès lors quelles sont mises au service d'une épreuve pratique " (Depraz, 2015, 136). Le " Je » acquiert donc du sens à partir du moment où il implique une expérience et une situation données. Nous pouvons ainsi voir dans la phénoménologie une pratique à la première personne dans une écriture qui laisse suggérer un Je générique et, donc, une perspective à la troisième personne. La lecture que nous avons désormais des textes du corpus husserlien prend pleinement son sens, et Natalie Depraz ajoute à cela qu'une lecture expérientielle des textes, qui suit une lecture plus traditionnelle, disons " conceptuelle " ou " herméneutique ", laisse entrevoir cette autre dimension de l'écriture phénoménologique à partir des exemples qui y sont donnés. Prévaut toujours l'idée d'une phénoménologie à/de la première personne qui, malgré son usage diversifié du pronom personnel « Je », situe son propos et l'incarne dans une situation donnée. Si, quand bien même elle échouait par moments à réaliser un tel besoin épistémologique, elle permet malgré tout au lecteur de s'interroger sur son rôle de donateur de sens. Il faut par conséquent distinguer deux types d'usage du " Je » comme indexical : 1) le premier, rhétorique, qui ne renvoie à aucune expérience située et à aucun sujet précis et incarné. Il servirait à parler de tout et de rien, dans une indistinction spatio-temporelle totale. 2) Le second usage est quant à lui la description d'une situation, d'un vécu d'une subjectivité incarnée, d'un " processus expérientiel ». Comme le mentionne Depraz en guise de conclusion à son article, "l'enjeu d'une phénoménologie authentiquement en première personne est profondément (auto-) éthique ", " une "éthique pratique" qui tient en cinq traits : 1) la fidélité au donné et à l'expression ;2) la précarité provisoire de la description ; 3) la confiance dans ce qui est obtenu ; 4) la dynamique du perfectionnement par l'exercice ; 5) la liberté intérieure grâce à la vigilance » (Depraz, 2015, 145-146). En quelques mots, il est nécessaire de prendre conscience du rôle de l'indexical " Je " afin de saisir en quoi la philosophie phénoménologique transcendantale peut nous égarer quant à l'expression même de l'expérience.

Mais plus que cela, nous pouvons nous interroger quant à la portée de la réduction transcendantale en tant qu'elle nous empêche de saisir la dimension expérientielle et vivante du sujet. Comme le note Renaud Barbaras, la réduction transcendantale et toute forme de retour aux fondements transcendantaux de la vie prennent précisément appui sur ce qui manque la vie : un «mouvement, engagé par Husserl, d'une "transcendantalisation" de la vie. Mais cette vie purement transcendantale, 
étrangère au monde, est une vie exténuée, une vie désertée par le vivant » (Barbaras, 2008, 9-10). A cela, nous pourrions répondre que Husserl, dans les Méditations cartésiennes, affirmait qu'une expérience transcendantale existe bel et bien (Hua I, II). Or, cette expérience se limite à l'examen du cogito transcendantalement réduit. En ce sens, il nest pas question d'une expérience mondaine ou psychologique, c'est-à-dire d'une expérience située pour un sujet singulier. Il s’agit précisément d'une expérience impersonnelle à la troisième personne. Et, si l’on se réfère une fois de plus aux propos de Natalie Depraz, nous pourrions nous demander si la voie de la psychologie ne serait pas davantage adéquate dans la caractérisation de la vie subjective que la voie cartésienne qui ne nous permet, finalement, que de découvrir l'antichambre de la subjectivité qu'est la sphère transcendantale : "Contrairement à la seule voie cartésienne dont la radicalité du point de départ, apodictiquement fondateur, est à la mesure de son étroitesse, de sa pauvreté voire de sa stérilité phénoménologique, [...] la voie de la psychologie permet de fonder la pertinence de l'intersubjectivité husserlienne, laquelle se soutient elle-même dans la configuration des trois voies " (Depraz, 1995, 25). Contrairement à la voie cartésienne de la réduction ou la voie du monde de la vie, la voie de la psychologie décrite par Husserl dans ses écrits relatifs à la Psychologie phénoménologique met en avant le caractère incarné du sujet, son vécu singulier. La voie de la psychologie situe le sujet de même que son expérience. Elle rend compte du caractère expérientiel de la vie subjective. Ce faisant, le risque de la réduction consiste en une transcendantalisation extrême qui surdétermine ce qu'elle s'efforce de nous faire voir, tandis que la voie de la psychologie rend possible une réduction qui nous permette de saisir à la fois la dimension intersubjective de la vie et les objectités sans recours à une surdétermination stérile. Par conséquent, le revirement génétique de la phénoménologie nous permet d'appréhender plus justement le domaine de la vie subjective en ce qu'il nous ouvre un horizon qui s'inscrit directement dans le domaine du concret. D'une phénoménologie statique encore trop proche de l'entreprise kantienne, Husserl s'est donc attelé à développer une phénoménologie de la vie qui va de pair avec un naturalisme phénoménologique.

\section{DU «NATURALISME PHENOMENOLOGIQUE » A LA NATURALISATION DE LA PHENOMENOLOGIE}

Parler de «naturalisme phénoménologique » peut étonner. En effet, n’est-ce pas là une transgression à l'égard de l'idéal de Husserl ? Cette expression a priori oxymorique nous sensibilise toutefois sur l'ambivalence des propos que tient Husserl au terme de son œuvre, en particulier dans le volume consacré aux Grenzprobleme relatifs 
aux questions portant sur la naissance, la mort et l'inconscient. Le naturalisme phénoménologique naît d'une lecture du corpus husserlien qui se veut pour le moins radicale, en ce que nous prenons Husserl aux mots, lorsque celui-ci traite de la naissance, de la vie, et de la mort du sujet transcendantal, etc., en d'autres termes de la nature $d u$ sujet. Davantage, cette lecture nous permet plus aisément de faire le lien entre son œuvre et les recherches actuelles qui consistent à naturaliser la phénoménologie. En nous inspirant des propos de Merleau-Ponty, nous pouvons concevoir l'ambivalence de la phénoménologie à partir du terme de nature : "La phénoménologie dénonce l'attitude naturelle et, en même temps, fait plus quaucune autre philosophie pour la réhabiliter " (Merleau-Ponty, 1995, 104). En soulignant cette difficulté interprétative inhérente à toute l'œuvre de Husserl, Merleau-Ponty met le doigt sur un aspect bien souvent mis sous silence : la phénoménologie en vient toujours, en dernière instance, à comprendre quelle est la vie du sujet - qu'il s'agisse de la vie mondaine ou de la vie transcendantale. Toutefois, si le sujet est capable de comprendre grâce à la réduction ce qu'est une vie qui n'est plus anonyme, il n'est pas, à vrai dire, de sujet qui pratique à temps complet la réduction. C'est pourquoi la vie doit être étudiée non seulement dans sa composante constitutive, c'est-à-dire à partir des modes de donation de sens propres au sujet méditant, mais aussi à partir de cette «activité passive » de la vie dans laquelle l'ego transcendantal n'est engagé que de façon latente. Dans cette optique, Merleau-Ponty a raison d'affirmer que la phénoménologie virevolte constamment entre deux attitudes, entre deux formes de vie, car sa visée d'une science idéale, dont le fondement est l'ego meditans, ne peut se passer d'un examen des modalités doxiques qui sont à l'origine d'un savoir toujours à disposition dont il faut actualiser le sens par la réduction. Une telle attitude suscite un questionnement de taille pour MerleauPonty qui aura certainement été l'un des premiers à mettre en lumière cette part méconnue de la phénoménologie : «Doit-on passer de la doxa à lépistémè, ou de la doxa à l'Urdoxa, à la doxa primordiale ? Si la philosophie commence par l'attitude naturelle, en sortira-t-elle jamais et, si elle en sort, pourquoi sort-elle ?» (Merleau-Ponty, 1995, 112-113). Nous répondrons à cette interrogation en avançant que celle-ci ne peut se passer de ces deux dimensions de la vie, quelles vont de pair lorsqu'il est question détablir une science dont le fondement est la vie du sujet : naturelle et transcendantale. La phénoménologie est ainsi une science fondatrice pour laquelle l'établissement d'une véritable épistémè dépend autant de l'Urdoxa que celle-ci de l'épistémè. Néanmoins l'Urdoxa n'est pas sans poser problème en ce que le phénoménologue est sans cesse dans l'obligation de rediriger son regard sur des formes de la vie qui dépassent la visée initiale de la phénoménologie, c'est-à-dire qui reposent sur une entente quasi mondaine de la vie. Voici la raison pour laquelle la notion de nature importe tant pour 
Husserl, en particulier dans ses leçons au sujet de la nature et de l'esprit ou dans le volume XLII des Husserliana. C'est notamment pour cela que cette même notion n'est jamais tout à fait la même dans son corpus, car elle dépasse la binarité idéalisme-réalisme, elle suppose un naturalisme phénoménologique qui puisse devenir une science globale de la vie de la conscience. Entendons par là que Husserl ne cesse de caractériser la nature tantôt comme ce qui est relatif au « temps et l'espace, les corps matériels, la forme, la position, le mouvement et la direction du mouvement, le changement, aussi l'action, la force, etc. » (Husserl, 2001, 10), tantôt comme ce qui est relatif à la dimension corporelle et mondaine du sujet. Cette nécessité d'une compréhension globale de la vie subjective peut se comprendre avec les propos de Merleau-Ponty: «Tout ce qui se passe ne s'explique pas par l'intériorité, ni par l'extériorité, mais par une chance, qui est la concordance entre ces deux données, et qui est assurée par la Nature » (MerleauPonty, 1995, 111). Il importe par conséquent de lier les termes de vie et de nature afin de comprendre de quelle manière la méthode pratiquée tardivement par Husserl peut être désignée comme un naturalisme phénoménologique.

Pourtant, dans l’optique dans une naturalisation de la phénoménologie, le terme de nature demeure problématique. En effet, si l’œuvre tardive de Husserl regorge de fines d'analyses permettant de conjoindre la phénoménologie aux sciences de la nature grâce au naturalisme phénoménologique, il n’entendait pas unifier de manière si significative sa méthode avec celle des sciences de la nature. Au contraire, n’a-t-il pas écrit une quantité non négligeable de papiers relatifs à ce refus ? Pour preuve, cette lettre adressée à Rickert en 1915: « Donc, je me sens, depuis une décennie, étroitement lié avec les responsables des écoles idéalistes allemandes, nous combattons comme des alliés contre le naturalisme de notre temps en tant qu'il est notre ennemi commun" (Husserl, 1994, 178). Plus largement, suite au tournant génétique de sa philosophie, ses écrits sur la psychologie phénoménologique récusent strictement toute entreprise naturaliste :

Lattitude thématique portant sur le monde et l'univers comme nature opère d'une certaine manière une césure avec toutes les attitudes dirigées sur ce qui est concret. [...] La pure considération du monde prive ainsi le monde d'esprit (entgeistigt), son intérêt est pareillement aveugle pour tout ce qui concerne le monde des hommes, pour ce qui a une signification pour quelque subjectivité que ce soit, et, alors, elle est naturellement aussi aveugle pour ce qui est subjectif, spécialement pour ce qui est humain, en général pour la vie de l'esprit, pour les personnes et les communautés personnelles, les valeurs, pour les beautés, le bon, etc. (Husserl, 1968, 382-383)

De telles considérations ne peuvent que renforcer le refus de toute naturalisation en ce quelles récusent l'attitude naturelle réductrice. Husserl a bel et bien traité de 
la nature à partir de thèmes aussi divers que la conscience, la vie, la naissance, la mort, en particulier dans les textes publiés dans le tome XLII des Husserliana consacré aux Grenzprobleme der Phänomenologie, mais le terme de nature demeure problématique dans le cadre d'un réductionnisme naturaliste. De la nature il y a deux ententes différentes dans le corpus phénoménologique, d'abord la nature comme ensemble de l'étantité matérielle dans laquelle nous nous mouvons et que nous étudions scientifiquement dans une perspective à la troisième personne, et comme nature du sujet, autrement dit, une nature constituée et constituante. Comprenons dès lors qu'il ne peut $\mathrm{y}$ avoir de naturalisation qui prenne appui sur la première entente de la nature - la nature comme matière, comme objet de science. La nature dans un sens strictement phénoménologique est relative à la vie du sujet constituant. En ce sens, la naturalisation est phénoménologique si elle n’a affaire quà la question de la constitution du sens. Qu'il s’agisse de données phénoménologiques ou de données scientifiques, il est nécessaire qu’elles convergent vers ce même objectif qui est de définir les qualités constitutives de la vie du sujet.

Le tournant génétique de la phénoménologie, c'est-à-dire l'avènement d'un questionnement tourné sur la vie du sujet en sa dimension concrète, mondaine et psychologique, permet de dépasser le dualisme que l'on pouvait imputer à la première forme de phénoménologie esquissée par Husserl. Il s’agit précisément de ce que nous nommons ici un naturalisme phénoménologique, pour ne pas dire une phénoménologie générative ou un empirisme transcendantal, comme le proposent Anthony Steinbock ou Natalie Depraz. Nous proposons de prendre Husserl au mot, et de reprendre le terme de nature tel qu'il l'a employé lorsqu'il sest interrogé sur la vie, la vocation, le monde de la vie, lêtre psycho-physique, etc. C'est grâce à ce type particulier d'analyse que ces thèmes trouvent une véritable explicitation phénoménologique, mais aussi un ancrage davantage mondain. En effet, Husserl s'applique à situer son propos dans son époque, en particulier lorsqu'il est question de l'anomalité, de la crise des sciences européennes, ou de l'attention singulière qu'il faut accorder aux phénomènes alors croissants de xénophobie et d'exclusion. La mise entre parenthèses de la thèse du monde nécessaire lors de la pratique de la réduction ne signifie nullement un oubli du monde dans lequel nous nous trouvons en tant que sujets incarnés liés dans une communauté intersubjective. Au contraire, les derniers textes nous indiquent qu'il y a un télos pratique de la phénoménologie qui ne se réduit pas à la dimension strictement transcendantale et immanente. En l'occurence, la Lebenswelt, contrairement à la sphère transcendantale fermée sur elle-même, constitue le lieu d'un Té̉os pour la raison en quête perpétuelle de sens. Tel que nous pouvons le lire dans le volume consacré à la Lebenswelt: « dans l'immanence il n’y a pas de télos pour la vie du Je » (Husserl, 2008, 
21). Par conséquent, il est important de noter que la phénoménologie husserlienne connaît un véritable revirement au terme de son élaboration. Pourtant, l'apport de la réduction phénoménologie et le recours à la dimension transcendantale du sujet, comme pierre angulaire de toute connaissance, ne doivent pas être négligés et Husserl nentend aucunement y renoncer. Seule la pratique de la réduction permet de rendre compte du rôle déterminant de la subjectivité dans le domaine de la science entendue en un sens large. Peut-être est-ce une façon de revoir ce que Husserl affirmait dans les derniers passages des Méditations cartésiennes: « toute analyse et toute théorie phénoménologique transcendantale $-\mathrm{y}$ compris la théorie de la constitution transcendantale du monde objectif [...] - peut être développée au niveau naturel dans l'abandon de l'attitude transcendantale ( (Husserl, 1947, 212). Mais nous ne devons pas oublier que seule une naturalisation phénoménologique mérite le titre de "naturalisation de la phénoménologie ", car elle s'appuie sur des méthodes entièrement nouvelles que Husserl a mises en lumière. « D’ailleurs, la partie de beaucoup la plus importante des recherches phénoménologiques appartient à une psychologie intentionnelle apriorique et pure (c'est-à-dire libérée de tout ce qui touche à la psycho-physiologie) » (Husserl, 1947, 231). Il faut à ce sujet se souvenir que Husserl, dès les leçons de 1923/24 et le tournant génétique de sa philosophie, sest attelé à décrire une réduction qui n'est plus uniquement cartésienne et solipsiste, mais qui prend en compte la situation incarnée du sujet. Phénoménologique, une telle réduction concerne la vie du sujet et respecte l'esthétique transcendantale développée par Husserl. Comme le dit Natalie Depraz,

on a là une structure, quelque chose qui respecte la consigne que donnait Husserl, lorsqu'il proposait la réduction et qui se situe sur le terrain de ce qui est formellement nommé le transcendantal. Cette voie permet aussi déviter l'opposition entre ce qui est de l'ordre du transcendantal, le structurel, et ce qui est de l'ordre du psychologisme. La voie de la psychologie respecte l'esthétique transcendantale de Husserl. La seule différence, c'est qu'il l'applique au domaine de la vie du sujet. (Thumser, 2017)

C’est dire si les liens de parenté entre la psychologie et la phénoménologie, les sciences naturelles de la subjectivité et la phénoménologie, sont étroits. Elles portent chacune sur le même objet, mais gagnent à pratiquer la réduction. Seulement, quoi que pût défendre Husserl à son époque, les paradigmes scientifiques qu'il niait alors n’en étaient quà leurs balbutiements. Aussi, faut-il nuancer son propos pour comprendre deux choses : d’abord que la science, en particulier ces nouvelles humanités appelées les sciences cognitives, connaît un renouveau sans commune mesure depuis une quarantaine d'années. De létude rudimentaire du système nerveux ou du comportement, les sciences de la subjectivité ont connu un revirement important lors- 
qu'elles ont pris en compte le domaine de la conscience humaine. Plus spécifiquement, la question de lobjectivité de la conscience peut être revisitée, car les sciences de la subjectivité tendent progressivement à dépasser le stade d'une dualité insurmontable en ce qui concerne la conscience comme objet de science, car elles létudient sans pour autant classifier la conscience comme un objet (Gegenstand). Du fait de ce renouveau épistémologique, il peut dorénavant advenir un nouveau commencement en phénoménologie, en ce quelle peut se joindre aux sciences cognitives dans un éclaircissement réciproque, pour aborder la question cardinale de la constitution du sens. Cela nous permettra sans nul doute de comprendre comment « on peut accepter les faits évidents de la physique - que le monde est entièrement constitué de particules physiques dans des champs de force - sans nier l'existence, au sein des caractéristiques physiques du monde, de phénomènes biologiques tels que des états de conscience qualitatifs internes et une intentionnalité intrinsèque " (Searle, 1995, 14). Il importe pour nous de conquérir ce nouveau continent scientifique pour pleinement appréhender de quelle façon la naturalisation de la phénoménologie entend atteindre une vue d'ensemble de cette constellation si bariolée que sont les vécus-de-conscience et leurs soubassements neurophysiologiques. Si la conscience peut bien entendu être étudiée à partir d'un examen de type neuroscientifique, nous pourrions néanmoins être étonnés de l'importance que peuvent avoir d'autres dimensions du vivant et du corps pour ce qui relève de la naturalisation, comme le cœur, le système nerveux entérique, etc.

\section{CONCLUSION}

Nous constatons, en fin de compte, que l'idéalisme transcendantal dont se parent la philosophie kantienne et la philosophie phénoménologique nest en rien limitatif. D’une part, parce qu'une approche statique va de pair avec une approche génétique, voire naturalisante, en ce que la mise en lumière des cadres a priori et universels à l'origine de toute forme de connaissance ne peut se passer d'une étude de l'inscription corporelle du sujet dans le monde intersubjectif. Sans cela, la philosophie demeurerait empêtrée dans une vision similaire à celle développée dans la Critique de la raison pure, c'est-à-dire dans une transcendantalisation exacerbée de la vie qui mène à une césure entre le phénoménal et le transcendantal, et qui nierait par la même occasion la vie en sa dimension globale empirico-transcendantale. D’autre part, létude de la phénoménologie husserlienne peut servir le chercheur en sciences cognitives en ce qu'il sera en mesure de comprendre la portée d'un retour à la sphère subjective. Ce faisant, toute forme d'entreprise scientifique pourra devenir pleinement phénoménologique. Plus encore, nous avons souhaité mettre l'accent sur l'ambivalence et la portée des textes 
tardifs de Husserl afin de montrer en quoi ces derniers sont en mesure d'apporter un éclaircissement aux sciences cognitives. Le naturalisme phénoménologique dont nous évoquions l'existence n'est pas une naturalisation au sens strict, c'est-à-dire une entreprise visant à formaliser le vivant d'un point de vue facto-logique, mais une première élaboration de type phénoménologique d'un questionnement plus vaste. En ce sens, les développements relatifs aux thèmes de l'empathie, de la naissance, de l'inconscient, de la conscience préréflexive ou de la mort sont des exemples qui peuvent contribuer à la réalisation d'une science globale de la vie subjective du fait qu'ils apportent un éclairage nouveau sur les modalités transcendantales et ontiques de la vie du sujet. Ce faisant, les sciences cognitives ont tout à gagner en s'inspirant de la phénoménologie husserlienne, mais il ne faut pas négliger la part transcendantale et expérientielle de la vie de sorte qu'une naturalisation pleinement phénoménologique puisse voir le jour et ne pas perpétrer une approche uniquement à la troisième personne. Il faut en effet que la vie du sujet soit interrogée non seulement à partir des cadres transcendantaux ou ontiques via ce que Jean-Luc Petit nomme également « une régression contre-transcendantale » (Petit, 2015, 265) vers les constituants du sens que sont les organes, mais qu'elle prenne avant tout en compte la dimension individuelle, psychologique et expérientielle de la vie.

\section{REFERENCES}

Barbaras, R. (2008). Introduction à une phénoménologie de la vie. Paris: Vrin.

Benoist, J. (1994). Autour de Husserl : l'ego et la raison. Paris: Vrin.

Berger, G. (1941). Le cogito dans la philosophie de Husserl. Paris: Aubier Éditions Montaigne.

Berthoz, A., \& Petit, J-L. (2006). Physiologie de l'action et phénoménologie. Paris: Odile Jacob.

Cobb-Stevens, R. (1998). Husserl et la philosophie analytique. Paris: Vrin.

Depraz, N. (1995). Transcendance et incarnation. Le statut de l'intersubjectivité comme altérité à soi chez Husserl. Paris: Vrin.

Depraz, N. (2015). Première, deuxième, troisième personne. Bucarest: Zeta Books.

Foessel, M. (2015). Kant et l'équivoque du monde. Paris: CNRS.

Husserl, E. (1947). Méditations cartésiennes. Paris: Vrin.

Husserl, E. (1968). Phänomenologische Psychologie. Vorlesungen Sommersemester 1925 (Hua IX). The Hague: Martinus Nijhoff.

Husserl, E. (1994). Briefwechsel, Band V: Die Neukantianer. The Hague: Kluwer.

Husserl, E. (2001). Natur und Geist: Vorlesungen Sommersemester 1927 (Hua XXXII). Dordrecht: Kluwer.

Husserl, E. (2008). Die Lebenswelt. Auslegungen der vorgegebenen Welt und ihrer Konstitution. Texte aus dem Nachlass (1916-1937). New York: Springer.

Kant, E. (1986). Opus postumum. Paris: PUF.

Kant, E. (1993). Anthropologie d'un point de vue empirique. Paris: GF.

Kant, E. (2006). Critique de la raison pure. Paris: GF. 
Kant, E. (2012). Prolégomènes à toute métaphysique future. Paris: Vrin.

Merleau-Ponty, M. (1995). La Nature, Cours du Collège de France. Paris: Seuil.

Patočka, J. (2016). Le monde naturel comme problème philosophique. Paris: Vrin.

Petit, J-L. (2015). De la neurodégénérescence motrice comme limitation interne du " pouvoir-faire ». In J. Rogozinki (Ed.), Les cahiers Philosophiques De Strasbourg. La naturalisation de la phénoménologie 20 ans après. Strasbourg: Presses Universitaires de Strasbourg.

Philonenko, A. (1969). L'oeuvre de Kant. Tome I. La philosophie pré-critique et La Critique de la Raison Pure. Paris: Vrin.

Searle, J. (1995). La redécouverte de l'esprit. Paris: Gallimard.

Thumser, J-D. (2017). Entretien avec Natalie Depraz: Autour de la cardiophénoménologie. Un Philosophe. Retreived from: https://unphilosophe.com/2017/10/09/entretien-avec-natalie-depraz-autour-de-la-cardiophenomenologie/ 